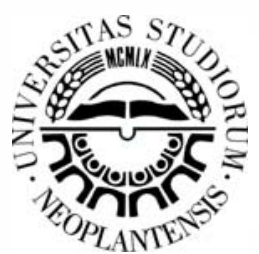

\title{
Performance Evaluation of the Effects of Post Weld Heat Treatment on the Microstructure, Mechanical and Corrosion Potentials of Low Carbon Steel
}

\author{
Isiaka Oluwole Oladele ${ }^{1 *}$, Davies Babatunde Alonge ${ }^{1}$, Timothy Olakunle Betiku ${ }^{1,2}$, Emmanuel $^{2}$ \\ Ohiomomo Igbafen ${ }^{1}$, Benjamin Omotayo Adewuyi ${ }^{1}$
}

${ }^{1}$ Department of Metallurgical and Materials Engineering, Federal University of Technology, Akure, Nigeria ${ }^{2}$ Université Grenoble Alpes, PHELMA, Grenoble Institute of Engineering, 38000 Grenoble, France.

\section{ABSTRACT}

\begin{abstract}
The effect of Post Weld Heat Treatment (PWHT) on the microstructure, mechanical and corrosion properties of low carbon steel have been investigated. The welding process was conducted on butt joint using Manual Metal Arc Welding (MMAW) techniques at a welding voltage of $23 \mathrm{~V}$ and welding current of $110 \mathrm{~A}$ with the use of E6013 and $3.2 \mathrm{~mm}$ diameter as filler material. Heat treatment through full annealing was carried out on the welded low carbon steel. The mechanical properties (hardness, impact toughness and tensile properties) of the AW and PWHT samples were determined. The microstructure of the AW and PWHT samples was characterized by means of an optical microscopy. Corrosion behavior of the sample was studied in3.5 wt.\% NaCl environment using potentiodynamic polarization method. The results showed that the AW samples has good combination of mechanical and corrosion properties. The microstructure revealed fine grains of pearlite randomly dispersed in the ferrite for the AW base metal (BM) sample while agglomerated and fine particle of epsilon carbide or cementite randomly dispersed on the ferritic phase of the heat affected zone (HAZ) and weld metal (WM), of the AW, respectively. The PWHT samples shows that the annealing process allow diffusion and growth of the fine grains into partial coarse grains of ferrite and pearlite which did not encourage improvement of the properties. Therefore, it was concluded that the welding parameters put in place during welding of the low carbon steel are optimum for quality weld.
\end{abstract}

Key words: low carbon steel; welding; post weld heat treatment; mechanical properties; microstructure; corrosion behaviour.

\section{$1 \quad$ INTRODUCTON}

Low carbon steel is an essential engineering material which have found different applications in marine applications, transportation, chemical processing, pipelines, mining, construction and metal-processing equipment due its availability, cost, mechanical properties, ease of fabrication and weldability. Engineering components has been joined through different processes such as welding, fastening, riveting, brazing. In other to reduce time for manufacturing, achieve weight reduction, improvement in mechanical properties and to ensure permanent joint, welding process has been adopted for many manufacturing processes. Welding is a crucial joining process due to high joint efficiency, simple set up and low cost of operation[1]. Manual Metal Arc Welding (MMAW) is a major arc welding process in which fusing of metal is done by heat from an electric arc that is sustained between the tip of a covered electrode and the surface of the base metal. The process is commonly adopted because of its low cost of equipment, faster deposition rates and simple set-up compared to gas tungsten arc welding[2]. However, during the welding process, most weld joints undergoes localized heating; this makes the temperature distribution to be non-uniform, and this leads to structural and metallurgical changes as the welding progresses along the joint [3]. The cycle of heating and cooling that occurs during the welding

* Corresponding author's.e-mail: wolesuccess2000@yahoo.com 
process affects the microstructure and surface composition of the welds and adjacent base metal[4]. This may occur because of solute segregation or coarsening of grains. Any weld design must aim at ensuring the integrity of the weld and effectively minimize the weld defects [5]. Therefore, PWHT can be employed as a means of minimizing segregation and modifying the microstructure of the weldment [6]. PWHT has been a viable option to restore the strength of joints by modification of the size, shape and distribution of the secondary strengthening particles [7].

Earlier work has studied the effect of PWHT on the mechanical properties of medium carbon steel, high strength steels stainless steels and some aluminium alloys. The effect of post heat treatment on the microstructures and mechanical properties of a submerged-arc-welded 304 stainless steel by [8]. The results obtained showed that after heat-treatment, the pre-existing strips in the base metal was dissolved into austenite and the delta-ferrite of the weld metal was transformed into austenite, leading to the microstructure close to the austenite single phase. Although the yield strength was lower than that of the asweld specimen due to the disappearance of hard phases and the relaxation of residual stress, the elongation became higher than that of the as-weld metal due to the compromising effect of strength and partially to the deformation induced martensitic transformation occurring during deformation. Dodo et al. [9] investigated the effect of post-weld heat treatment on the microstructure and mechanical properties of arc welded medium carbon steel using the shielded metal arc welding technique and, thereafter, heat treated by annealing, normalizing and quench hardening in water. The results of the optical microscopic test show that fine grains of pearlite in ferrite were obtained in normalized samples and martensite was also observed in quenched samples. On the other hand, mechanical property tests indicated that normalized welded specimens gave good combination of mechanical properties. The effects of post weld heat treatments on the microstructure and mechanical properties of dissimilar weld of super-martensitic stainless steel using gas tungsten arc welding (GTAW) with super-duplex stainless steel filler metal was studied by [10]. The result obtained shows that the impact toughness of the weld metal decreased with the increase of time of heat treatment, due to intermetallic precipitation. Also, it was found that optimum mechanical properties can be obtained with heat treatment for 30 minutes at $650^{\circ} \mathrm{C}$.

Research is ongoing to unify the microstructure of weldment through PWHT thereby reducing residual stress, ensuring dimensional stability and reducing heat affected zone of weldment to enhance properties and performance of welded joints of steel in application. In the light of this, several papers have been published on the effects of PWHT on the properties of different types and grades of steel. In this study, investigation of effect of PWHT on the mechanical and corrosion properties of manual-metal arc welded joint of low carbon steel in sea water was carried out.

\section{MATERIALS AND METHODS}

Low carbon steel of $6 \mathrm{~mm}$ thickness and mild steel electrode E6013 was used for this research. The chemical composition of the low carbon steel and electrode E6013 was shown in the Table 1 and 2.

Table 1- Chemical composition of low carbon steel (\%)

\begin{tabular}{ccllccc}
\hline Elements (\%) & $\mathrm{Fe}$ & $\mathrm{C}$ & $\mathrm{Mn}$ & $\mathrm{Si}$ & $\mathrm{P}$ & $\mathrm{Ni}$ \\
\hline Compositions & 98 & 0.157 & 0.279 & 1.28 & 0.0213 & 0.022 \\
\hline
\end{tabular}

Table 2 - Chemical composition of mild steel electrode E6013 (\%)

\begin{tabular}{ccccccc}
\hline Elements (\%) & $\mathrm{Fe}$ & $\mathrm{C}$ & $\mathrm{Mn}$ & $\mathrm{Si}$ & $\mathrm{P}$ & $\mathrm{Ni}$ \\
\hline Compositions & balance & 0.12 & 0.3 & 0.35 & 0.04 & 0.035 \\
\hline
\end{tabular}

The low carbon steel was cut into dimensions $40 \times 50 \times 6 \mathrm{~mm}$ with two pieces joined together through butt joint using MMAW machine techniques at a welding voltage $23 \mathrm{~V}$ and welding current of $110 \mathrm{~A}$ with the use of E6013 and $3.2 \mathrm{~mm}$ diameter as filler material. After welding, part of the samples was subjected to PWHT. PWHT was carried out on the samples by heating them to a temperature $850{ }^{\circ} \mathrm{C}$ for 1 hour and allowed to cool in the furnace. Afterwards, mechanical, microstructural examination and corrosion test were carried out on the BM, HAZ and WM of the AW and PWHT samples.

\subsection{Mechanical and corrosion tests}

Tensile test was performed on the sample according to ASTM D638 shown in Figure 1 (a) using Universal Testing Machine with the ends of test sample fixed into the grips connected to a straining device and to a load measuring device. Once the test sample fractured the result will be generated by the computer software.

Izod impact testing was carried out on the sample according to ASTM D256 shown in Figure 1 (b) using Honfield Balance Impact Testing Machine. Prior to mounting on the machine, the test sample was notched to a depth of $2 \mathrm{~mm}$ with $\mathrm{V}$-shaped hand file. The notched test sample was mounted on the impact testing machine, which was operated to apply a constant impact force on the test sample. The impact strength was read off the calibrated scale on the impact testing machine.

A digital Vickers micro-hardness tester, Indentec Hardness Testing Machine was used to measure the hardness of the BM, HAZ and WM across the welded joint of the samples according to ASTM E92-17 at an interval of $5 \mathrm{~mm}$ from the WM as shown in Figure 1 (c). Corrosion test of the BM, HAZ and WM of both the AW and PWHT samples was carried out using AUTOLAB potentiodynamic polarization test in sea water containing 3.5 wt. $\% \mathrm{NaCl}$ solution.

\subsection{Microstructural analysis}

Weldment of both the AW and PWHT samples which comprises of the BM, HAZ and WM were ground with different grades of emery papers and polished with selvyt 
cloth swamped with solution of $0.5 \mu \mathrm{m}$ Silicon carbide until a mirror-like surface was attained. The mirror-like surface was etched in 2\% NITAL (2\% Nitric acid and $98 \%$ of Ethyl Alcohol) after which it was washed and dried. The microstructure of the weldment was viewed under the Accu-scope optical microscope.

a)

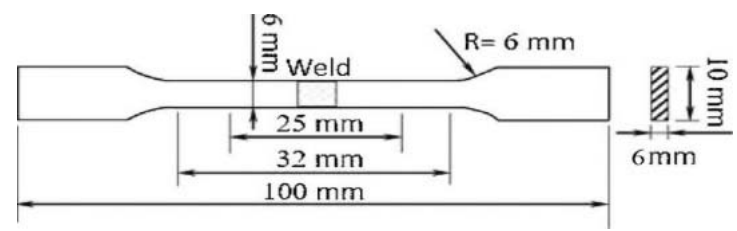

b)

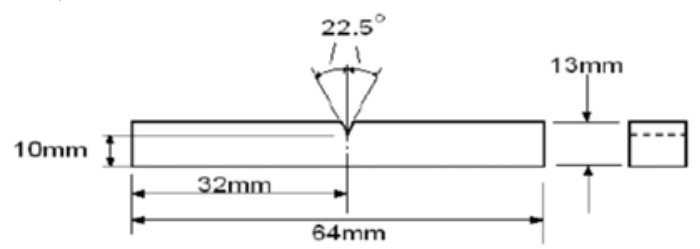

c)

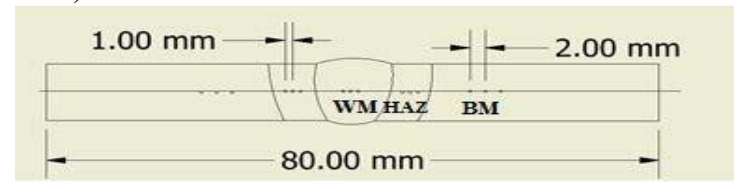

Fig. 1 Test samples (a) Tensile test specimen; (b )Impact test specimen; (c) Hardness test specimen.

\section{RESULTS AND DISCUSSION}

\subsection{Microstructural examination}

The optical micrographs of the weldment of the AW and PWHT samples were as shown in plate 1 . The micrograph in plate 1 (a) shows the microstructure of the BM of the AW sample. It was observed that it consists of the ferritic and pearlitic phase. The pearlitic phase are fine and randomly dispersed throughout the ferritic phase. After the BM of the AW sample has been subjected to full annealing operation, micrograph on Fig. 2 (d) was obtained which is the microstructure of BM of the PWHT sample. It was observed that the amount of dispersed pearlite present tends to be reduced. This occurred as aresult of diffusion and solubility of carbon atoms into the ferritic phase at higher temperature $\left(850^{\circ} \mathrm{C}\right)$ above its lower critical temperature which the sample was subjected.

The micrograph on Fig. 2(b) shows the microstructure of the HAZ of AW sample. From the microstructure, it was observed that there is agglomeration of pearlitic phase which are randomly dispersed in the ferritic phase. This could be as result of heat of welding which tends to alter the microstructure of the BM to create a region of HAZ. Since the welding heat is not constant, it does not allow uniform diffusion of the carbon atoms in the ferritic phase but rather short and random diffusion of carbon atoms which make them to agglomerate randomly forming pearlitic phase in the ferritic phase. After the HAZ of the AW sample has been subjected to full annealing, micrograph on Fig. 2 was obtained. The agglomerated pearlitic phase dissolved thereby making the carbon atoms to diffuse uniformly in the ferritic phase forming coarse ferrite structure, widmanstatten ferrite and some colonies of pearlite [11].

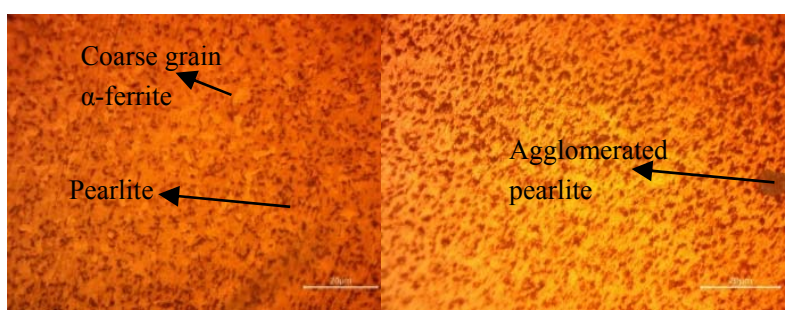

(a)

(b)

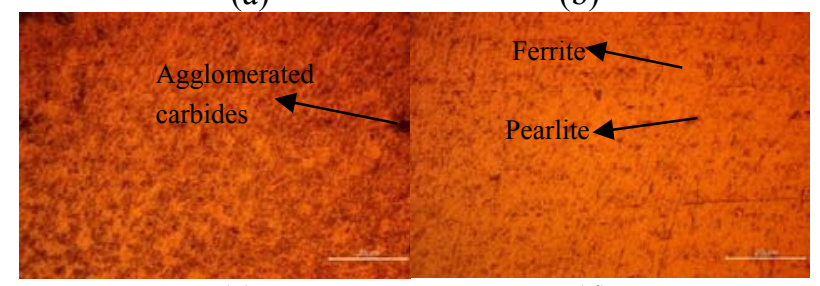

(c)

(d)

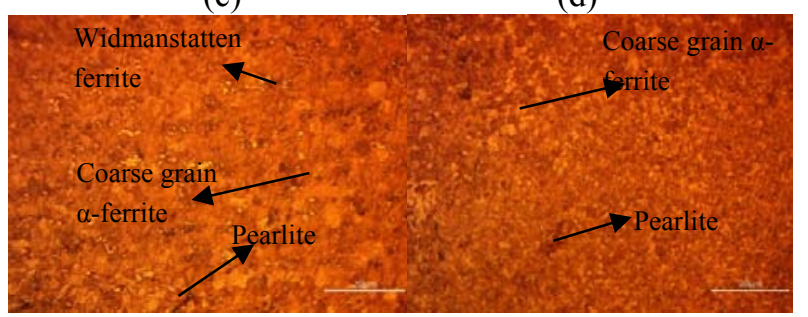

(e)

(f)

Fig 2 Optical micrographs of weldment of $A W$ and PWHT samples (a)AW of BM; (b) AW of HAZ; (c) AW of WM; (d) PWHT of BM; (e) PWHT of HAZ; (f) PWHT of WM.

The micrograph on Fig. 2 (c) represents the microstructure of WM of the AW sample. From the microstructure, it was observed that after welding, there were formation of refined grains of polygonal pearlite structure and agglomerated carbide[12] which are randomly dispersed across the ferritic phase. After full annealing of the WM of the AW sample, micrograph on Fig. 2 (f) was obtained. It was observed that the annealing process allow diffusion and partial growth of the fine grains into coarse grains of ferrite and pearlite.

\subsection{Mechanical properties}

The tensile properties which include tensile strength and yield strength of the joints in the AW, PWHT and as received (AR) samples are as shown in the Fig.2 and 3.

In Fig.2, the response of the steels revealed that AW sample seems to possess the best tensile properties. The ultimate tensile strength and strain was seen to be very high compared to other samples. It was observed that the sample was able to sustain the highest amount of stress to plastic deformation compared to the AR and PWHT 
samples. Therefore, the welding operation led to increment in the tensile properties of the welded sample while the heat treatment operation did not improve the tensile properties of the sample compared to the asreceived sample.

From Figure 3, the ultimate tensile strength and yield strength of the AW sample was 154.44 and $21.94 \mathrm{MPa}$, respectively while for the AR sample, the values are 117.30 and $32.32 \mathrm{MPa}$, respectively. It was noticed from the results that there was an improvement in the ultimate tensile strength of the welded sample compared to the as received sample that culminated to about $31.66 \%$. However, the yield strength was not enhanced which implies that the structural modification attained by the welding process could only aid this feat. This could be attributed to the fact that yield strength depended strongly

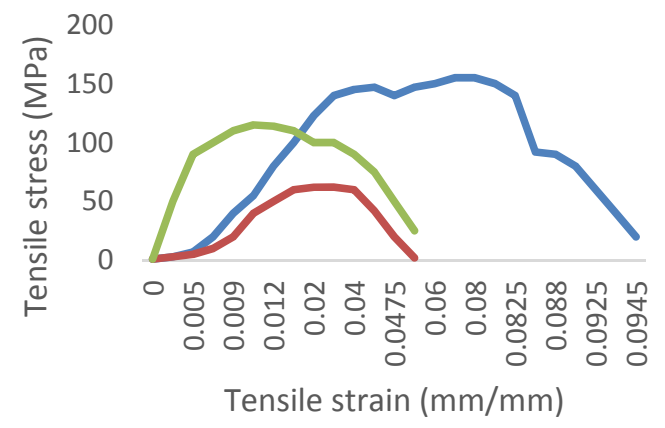

$\longrightarrow$ AW $\longrightarrow$ PWHT As received

Fig. 2 Tensile stress-strain curves for the welded mild steels and the as received sample.

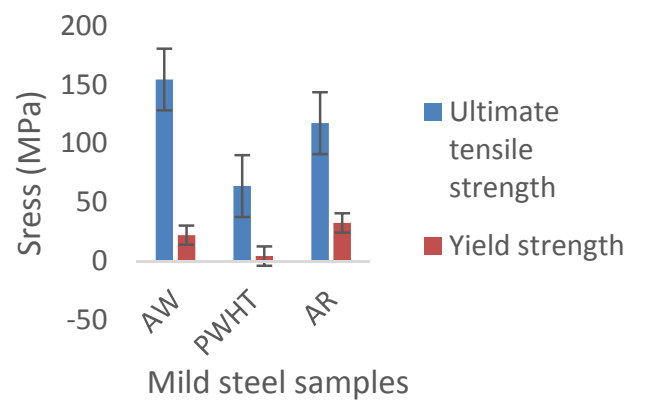

Fig. 3 Tensile strength of the welded mild steels and the as received sample.

on grain growth [13] which leads to decrease in the grain boundaries and presence of interrmetallic compounds formed in the weld metal which plays a significant role in affecting the yield strength of the sample.

Considering the effect of post weld heat treatment on the tensile strength, it was observed that the tensile strength and yield strength of the AW sample was greatly reduced by 77.33 and $44.95 \%$, respectively. The reduction in the tensile properties after post weld heat treatment may occur due to the full annealing operation which helped to relieve the residual stress induced by eliminating and redistributing dislocations at the region of the weld joint which are heavily distorted as a result of the welding process [14]. However, since the heat treatment was able to relief stress, this could also be relevant when considering the effect on the performance over a long period of time in service environment.

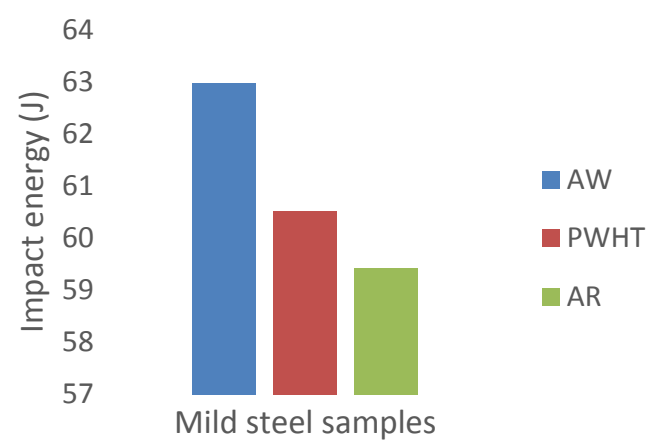

Fig. 4 Impact strength of the welded mild steels and the as received sample.

Figure 4 shows the results of the impact test for the AW, PWHT and AR samples. The welding process was noticed to improve the impact performance of the steels in both AW and PWHT conditions. From the results, it was observed that the impact strength of the AW and PWHT samples are greater than that of the as-received sample with the AW sample having the highest impact strength of about 62.97 J. However, after subjecting the AW sample to post weld heat treatment, its impact strength was reduced by about $4.09 \%$ and was with a value of about $60.52 \mathrm{~J}$. This could due to relief of internal stresses (residual stresses) due to recrystallization process that could possibly be taking place during the annealing operation which leads to decrease in the impact strength of the PWHT sample [15]. Contrary to the effect of post weld heat treatment on the tensile properties in Figures 2 and 3 , it was revealed here that by welding the steel and carrying out post weld heat treatment on the steels, the impact property can be improved relative to the as received which has a value of about $59.43 \mathrm{~J}$. Therefore, for long period application, in which impact strength is of great importance, post weld heat treatment should be considered since this would have helped in reducing internal stress. The effect of internal stress on weld joint is early failure which is not desirable.

It was observed from the results obtained in Figure 5, that the post weld heat treatment influenced the variations in hardness value across the weldment of the AW sample. The average hardness values at the weld metal for both AW and PWHT samples were 73.80 and $72.70 \mathrm{HV}$ while the average hardness values at the heat affected zones (HAZ) were 71.80 and $70.95 \mathrm{HV}$, respectively. The enhanced hardness in the WM is attributed to the annealing process which allowed diffusion of the agglomerated carbides and partial growth of fine grains of pearlite structure in WM. The reduction in hardness values across the weldment of AW sample after PWHT was due to reduction in the residual stresses induced in the AW sample during welding operation [3]. 
Figure 6 shows the Potentiodynamic polarization curves of the weldment of AW and PWHT samples immersed in sea water $3.5 \mathrm{wt} \% \mathrm{NaCl}$ solution environment. It was observed that the samples displayed similar polarization and passivity characteristics. However, the corrosion current densities (Icorr) and corrosion potentials (Ecorr) indicate clear distinct corrosion behaviour between the AW and the PWHT samples. It was observed from the plots that the corrosion current densities were more severe for the PWHT samples in comparison with the AW samples. This indicates that the AW samples are more corrosion resistance than the PWHT sample in the environment. The corrosion rates of the weldment of the AW weld metal sample was greatly increased after post weld heat treatment by $99.14,67.29$ and $55.70 \%$ for BM, HAZ and WM, respectively. Similarly, the increase in the values of the corrosion current densities (Icorr) and corrosion potentials (Ecorr) of the weldment after post weld heat treatment increased its susceptibility to corrosion. The high Icorr and Ecorr values of the weldment of the PWHT sample could be that annealed sample may not have reasonable tolerance for corrosion in harsh environment [16].

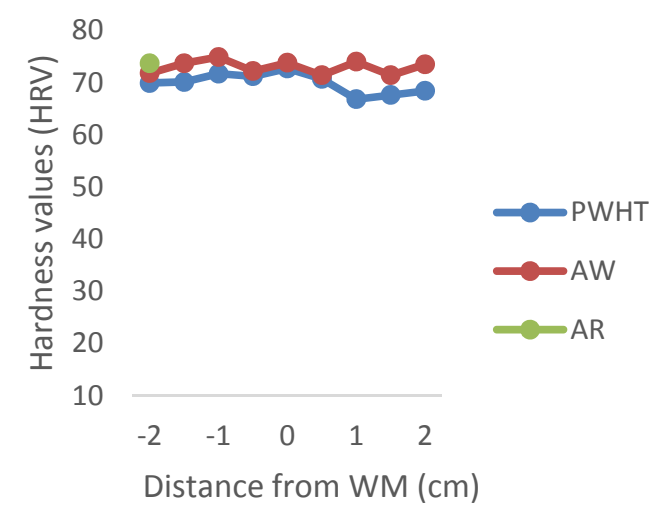

Fig. 5 Variations in hardness value across the weld of AW and PWHT samples.

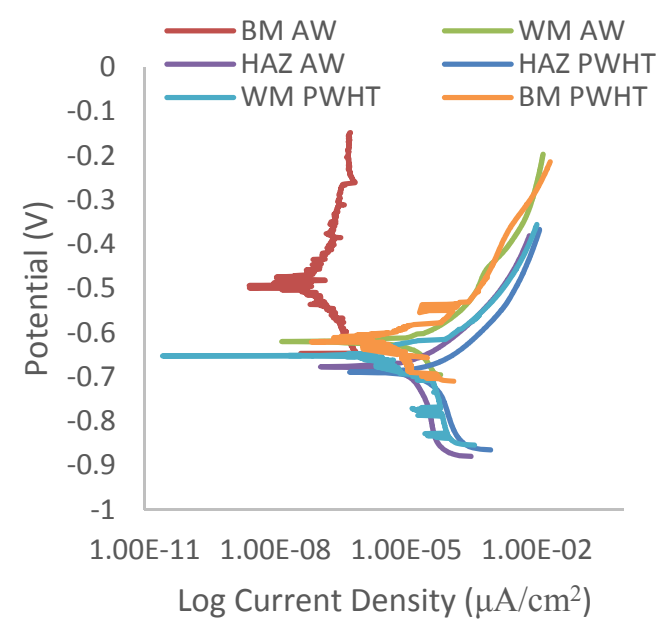

Fig. 6 Potentiodynamic polarization curves of the as weld and post weld heat treated samples in sea water containing $3.5 \mathrm{wt} \% \mathrm{NaCl}$ solution.

\section{CONCLUSIONS}

The investigation revealed that, selection of appropriate welding variables and post weld heat treatment for steels of difference sizes is very essential. The tensile, impact and hardness properties were enhanced after the welding of the steel compared to the as-received and post weld heat treated samples.

The corrosion resistance of the as-weld sample was higher than that of the as-received and post weld heat treated samples which imply that the welding parameters as well as the welding techniques put in place were optimum. The post weld heat treatment seems not to be necessary for welding process and variables selected for the welding of this steel.

\section{REFERENCES}

[1] Talabi, S. I., Owolabi, O. B., Adebisi, J. A., Yahaya, T. (2014). Effect of welding variables on mechanical properties of low carbon steel welded joint. Advances in Production Engineering \& Management, 9(4), 181-186.

[2] Oladele, I. O., Betiku, O. T., Fakoya, M. B. (2017). Effect of post weld heat treatment on the mechanical and corrosion. Leonardo Electronic Journal of Practices and Technologies, 30, 75-86.

[3] Masubuchi, K., Blodgett, O. W., Matsui, S., Ruud, C. O., Tsai, C. L. (2001). Welding Handbook. (9th ed.). American Welding Society. Miami.

[4] AbdRazak, N. A., Ng, S. S. (2014). Investigation of Effects of Mig Welding on Corrosion Behaviour of AIsI 1010 Carbon Steel. Journal of Mechanical Engineering and Sciences, 7(2), 1168-1178.

[5] Owolabi, O. B., Aduloju, S. C., Metu, Chidiebere, S., Chukwunyelu, C. E., Okwuego, E. C. (2016). Evaluation of the effects of welding current on mechanical properties of welded joints between mild steel and low carbon steel. AmericanJournal of Materials Science and Application, 4(1), 1-4.

[6] Mohd, N. S., Abdul, R., Mamat, S., Ahmad, M. I. (2016). Review on Welding Residual Stress. ARPN Journal of Engineering and Applied Sciences, 11(9), 6166-6175.

[7] Funderburk, R. S. (1998). Key Concepts in Welding Engineering. Welding Innovation, 15(2), 1-2.

[8] Nam, T.H.,Eunsol, A. N., Byung, J. K., Sunmi, S., Won, S. K., Nokeun, P.,Namhyun, K., Jong. B. J.(2018). Effect of Post Weld Heat Treatment on the Microstructure and Mechanical Properties of a Submerged-Arc-Welded 304 Stainless Steel. Molecular diversity preservation international, 8(26), 1-13.

[9] Dodo, M. R., Ause, T., Adamu, M. A., Ibrahim, Y. M. (2016). Effect of Post-Weld Heat Treatment on The Microstructure and Mechanical Properties of Arc Welded Medium Carbon Steel. Nigerian Journal of Technology, 35(2), 337-343. 
[10] Tavares, S. M., Rodrigues, C. R., Pardal, J. M., Barbosa, E. S., Gomes, H. F., (2014). Effects of Post Weld Heat Treatments on the Microstructure and Mechanical Properties of Dissimilar Weld of Supermartensític Stainless Steel. Materials Research, 17(5), 1336-1343.

[11] Boumerzoug, Z., Derfouf, C., Baudin, T. (2010). Effect of Welding on Microstructure and Mechanical Properties of an Industrial Low Carbon Steel. Scientific research, 2, 502-506.

[12] Datta, R., Veeraraghavan, R., Rohiraa, K. L. (2009). Weldability Characteristic of Torr and CorrosionResistant TMT Bar Using SMAW Process. Journal of Material Engineering and Performance, 11(2), 369-375.

[13] Guo, Y., Hu, J., Li, J., Jiang, L., Liu, T., Wu, Y. (2014). Effects of Annealing Temperature on the Mechanical and Corrosion Behavior of a Newly
Developed Novel Lean Duplex Stainless Steel. Materials, 7, 6604-6619

[14] Olawale, J. O., Ibitoye, S. A., Oluwasegun, K. M., Shittu, M. D., Ofoezie, R. C. (2012). Correlation between Process Variables in Shielded Metal-Arc Welding (SMAW) Process and Post Weld Heat Treatment (PWHT) on Some Mechanical Properties of Low Carbon Steel Welds. Journal of Minerals and Materials Characterization and Engineering, 11, 891-895.

[15] Raji, A. N., Oluwole, O. O. (2012). Effect of Soaking Time on the Mechanical Propertties of Annealed Cold-Drawn Low Carbon Steel. Materials Sciences and Applications, 3, 513-518.

[16] Seidu, S. O., Kutelu, B. J. (2013). Effect of Heat Treatments on Corrosion of Welded Low Carbon Steel in Acid and Salt Environment. Journal of Minerals and Materials Characterization and Engineering, 1, 95-100. 